\title{
Construction of Dominating Sets of Certain Graphs
}

\author{
Saeid Alikhani ${ }^{1}$ and Yee-Hock Peng ${ }^{2}$ \\ ${ }^{1}$ Department of Mathematics, Yazd University, Yazd 89195-741, Iran \\ ${ }^{2}$ Department of Mathematics, Universiti Putra Malaysia, 43400 Serdang, Malaysia \\ Correspondence should be addressed to Saeid Alikhani; alikhani206@gmail.com
}

Received 15 November 2012; Accepted 1 February 2013

Academic Editor: Wai Chee Shiu

Copyright (C) 2013 S. Alikhani and Y.-H. Peng. This is an open access article distributed under the Creative Commons Attribution License, which permits unrestricted use, distribution, and reproduction in any medium, provided the original work is properly cited.

Let $G$ be a simple graph. A set $S \subseteq V$ is a dominating set of $G$, if every vertex in $V \backslash S$ is adjacent to at least one vertex in $S$. We denote the family of dominating sets of a graph $G$ with cardinality $i$ by $\mathscr{D}(G, i)$. In this paper we introduce graphs with specific constructions, which are denoted by $G(m)$. We construct the dominating sets of $G(m)$ by dominating sets of graphs $G(m-1)$, $G(m-2)$, and $G(m-3)$. As an example of $G(m)$, we consider $\mathscr{D}\left(P_{n}, i\right)$. As a consequence, we obtain the recursive formula for the number of dominating sets of $G(m)$.

\section{Introduction}

Let $G=(V, E)$ be a graph of order $|V|=n$. For any vertex $v \in V$, the open neighborhood of $v$ is the set $N(v)=\{u \in$ $V u v \in E\}$, and the closed neighborhood is the set $N[v]=$ $N(v) \cup\{v\}$. For a set $S \subseteq V$, the open neighborhood is $N(S)=$ $\bigcup_{v \in S} N(v)$, and the closed neighborhood is $N[S]=N(S) \cup S$. A set $S \subseteq V$ is a dominating set if $N[S]=V$, or equivalently, every vertex in $V \backslash S$ is adjacent to at least one vertex in $S$. The domination number $\gamma(G)$ is the minimum cardinality of a dominating set in $G$, and the family of $\gamma$-sets is denoted by $\Gamma(G)$. For a detailed treatment of this parameter, the reader is referred to [1]. Let $\mathscr{D}(G, i)$ be the family of dominating sets of a graph $G$ with cardinality $i$, and let $d(G, i)=|\mathscr{D}(G, i)|$. The domination polynomial $D(G, x)$ of $G$ is defined as $D(G, x)=$ $\sum_{i=\gamma(G)}^{|V(G)|} d(G, i) x^{i}$, where $\gamma(G)$ is the domination number of $G$.

The domination polynomial of a graph has been introduced by Alikhani in his Ph.D. thesis [2]. More recently it has been investigated with respect to special graphs, zeros, and application in network reliability; see [2-9].

Obviously study of the dominating sets of graphs is a method for finding the coefficients of the domination polynomial of graphs. Authors studied the construction of dominating sets of some families of graphs to study their domination polynomials; see [10-13]. In this paper we would like to study some further results of this kind.
In the next section we introduce graphs with specific construction which is denoted by $G(m)$. As examples of these graphs, in Section 3 we study the dominating sets of paths and some other graphs. As a consequence, we give a recurrence relation for $|\mathscr{D}(G(m), i)|$ and $D(G(m), x)$.

As usual we use $\lceil x\rceil,\lfloor x\rfloor$ for the smallest integer greater than or equal to $x$ and the largest integer less than or equal to $x$, respectively. In this paper we denote the set $\{1,2, \ldots, n\}$ simply by $[n]$.

\section{Dominating Sets of Graphs $G(m)$}

A path is a connected graph in which two vertices have degree 1 , and the remaining vertices have degree 2 . Let $P_{n}$ be the path with $V\left(P_{n}\right)=[n]$ and $E\left(P_{n}\right)=\{(1,2),(2,3), \ldots,(n-1, n)\}$; see Figure 1(a).

In this section we introduce graphs with specific construction and study their dominating sets. Let $P_{m+1}$ be a path with vertices labeled by $y_{0}, y_{1}, \ldots, y_{m}$, for $m \geq 3$. Let $G(m)$ be a graph obtained from $G$ by identifying a vertex of $G$ with an end vertex $y_{0}$ of $P_{m+1}$. For example, if $G$ is a path $P_{2}$, then $G(m)=P_{2}(m)$ is a path $P_{m+2}$.

We need some lemmas and theorems to obtain main results in this section. 


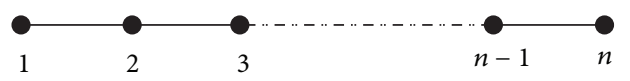

(a)

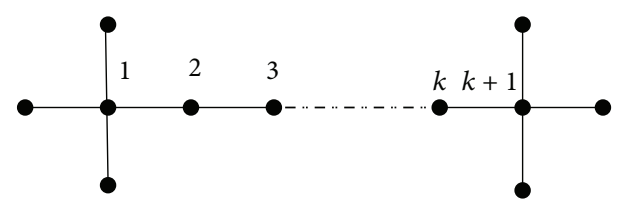

(b)

Figure 1: (a) The path $P_{n}$; (b) graph $G$ contains a simple path of length $k$.

Lemma 1. $\mathscr{D}(G(m), i)=\emptyset$ if and only if $i>|V(G(m))|$ or $i<\gamma(G(m))$.

Proof. It follows from the definition of dominating set of graph.

We recall the following theorem.

Theorem 2 (see [14]). If $e \in E(G)$, then $\gamma(G-e)-1 \leq \gamma(G) \leq$ $\gamma(G-e)$.

The following lemma follows from Theorem 2.

Lemma 3. For any $m \in N, \gamma(G(m-1)) \leq \gamma(G(m)) \leq \gamma(G(m-$ 1)) +1 .

Lemma 4 (see [15, page 371]). The domination number of path is $\gamma\left(P_{n}\right)=\lceil n / 3\rceil$.

A simple path is a path where all its internal vertices have degree two. In Figure 1(b), we have shown a graph $G$ which contains a simple path of length $k$ with vertices labeled $1, \ldots, k, k+1$. The following lemma follows from Lemma 4 .

Lemma 5. If a graph $G$ contains a simple path of length $3 k-1$, then every dominating set of $G$ must contain at least $k$ vertices of the path.

Lemma 6. If $Y \in \mathscr{D}(G(m-4), i-1)$, and there exists $x \in$ $\left\{y_{1}, y_{2}, \ldots, y_{m}\right\}$, such that $Y \cup\{x\} \in \mathscr{D}(G(m, i))$, then $Y \in$ $\mathscr{D}(G(m-3), i-1)$.

Proof. Suppose that $Y \notin \mathscr{D}(G(m-3), i-1)$. Since $Y \in$ $\mathscr{D}(G(m-4), i-1), Y$ contains at least one vertex labeled $y_{m-5}$ or $y_{m-4}$. If $y_{m-4} \in Y$, then $Y \in \mathscr{D}(G(m-3), i-1)$, a contradiction. Hence, $y_{m-5} \in Y$. But then in this case, $Y \cup\{x\} \notin \mathscr{D}(G(m), i)$, for any $x \in\left\{y_{1}, y_{2}, \ldots, y_{m}\right\}$, also a contradiction.

Lemma 7. (i) If $\mathscr{D}(G(m-1), i-1)=\mathscr{D}(G(m-3), i-1)=\emptyset$, then $\mathscr{D}(G(m-2), i-1)=\emptyset$.

(ii) If $\mathscr{D}(G(m-1), i-1) \neq \emptyset, \mathscr{D}(G(m-3), i-1) \neq \emptyset$ then, $\mathscr{D}(G(m-2), i-1) \neq \emptyset$.

(iii) If $\mathscr{D}(G(m-1), i-1)=\mathscr{D}(G(m-2), i-1)=\mathscr{D}(G(m-$ $3), i-1)=\emptyset$, then $\mathscr{D}(G(m), i)=\emptyset$.
Proof. (i) Since $\mathscr{D}(G(m-1), i-1)=\mathscr{D}(G(m-3), i-1)=\emptyset$, by Lemmas 1 and $3, i-1>|V(G(m-1))|$ or $i-1<\gamma(G(m-3))$. In either case we have $\mathscr{D}(G(m-2), i-1)=\emptyset$.

(ii) Suppose that $\mathscr{D}(G(m-2), i-1)=\emptyset$, so by Lemma 1, we have $i-1>|V(G(m-2))|$ or $i-1<\gamma(G(m-2))$. If $i-1>|V(G(m-2))|$, then $i-1>|V(G(m-3))|$, and hence, $\mathscr{D}(G(m-3), i-1)=\emptyset$, a contradiction. So we have $i-1<$ $\gamma(G(m-2), i-1)$, and hence, $\mathscr{D}(G(m-1), i-1)=\emptyset$, also a contradiction.

(iii) Suppose that $\mathscr{D}(G(m), i) \neq \emptyset$. Let $Y \in \mathscr{D}(G(m), i)$, then at least one vertex labeled $y_{m}$ or $y_{m-1}$ is in $Y$. If $y_{m} \in Y$, then by Lemma 5 , at least one vertex labeled $y_{m-1}, y_{m-2}$ or $y_{m-3}$ is in $Y$. If $y_{m-1} \in Y$ or $y_{m-2} \in Y$, then $Y-\left\{y_{m}\right\} \in$ $\mathscr{D}(G(m-1), i-1)$, a contradiction. If $y_{m-3} \in Y$, then $Y-\left\{y_{m}\right\} \in \mathscr{D}(G(m-2), i-1)$, a contradiction. Now suppose that $y_{m-1} \in Y$, then by Lemma 5 , at least one vertex labeled $y_{m-2}, y_{m-3}$ or $y_{m-4}$ is in $Y$. If $y_{m-2} \in Y$ or $y_{m-3} \in Y$, then $Y-\left\{y_{m-1}\right\} \in \mathscr{D}(G(m-2), i-1)$, a contradiction. If $y_{m-4} \in Y$, then $Y-\left\{y_{m-1}\right\} \in \mathscr{D}(G(m-3), i-1)$, a contradiction. Therefore $\mathscr{D}(G(m), i)=\emptyset$.

Lemma 8. Suppose that $\mathscr{D}(G(m), i) \neq \emptyset$, then

(i) $\mathscr{D}(G(m-1), i-1)=\mathscr{D}(G(m-2), i-1)=\emptyset$ and $\mathscr{D}(G(m-3), i-1) \neq \emptyset$ if and only if $\gamma(G(m-3))+1 \leq$ $i<\gamma(G(m-2))+1$,

(ii) $\mathscr{D}(G(m-2), i-1)=\mathscr{D}(G(m-3), i-1)=\emptyset$ and $\mathscr{D}(G(m-1), i-1) \neq \emptyset$ if and only if $i=|V(G(m))|$,

(iii) $\mathscr{D}(G(m-1), i-1)=\emptyset, \mathscr{D}(G(m-2), i-1) \neq \emptyset$ and $\mathscr{D}(G(m-3), i-1) \neq \emptyset$ if and only if $\gamma(G(m-2))+1 \leq$ $i<\gamma(G(m-1))+1$,

(iv) $\mathscr{D}(G(m-1), i-1) \neq \emptyset, \mathscr{D}(G(m-2), i-1) \neq \emptyset$ and $\mathscr{D}(G(m-3), i-1)=\emptyset$ if and only if $i=|V(G(m))|-1$,

(v) $\mathscr{D}(G(m-1), i-1) \neq \emptyset, \mathscr{D}(G(m-2), i-1) \neq \emptyset$ and $\mathscr{D}(G(m-3), i-1) \neq \emptyset$ if and only if $\gamma(G(m-1))+1 \leq$ $i \leq|V(G(m))|-2$.

Proof. (i) $(\Rightarrow)$ Since $\mathscr{D}(G(m-1), i-1)=\mathscr{D}(G(m-2), i-1)=\emptyset$, by Lemmas 1 and 3, we have $i-1>|V(G(m))|-1$ or $i-1<$ $\gamma(G(m-2))$. If $i-1>|V(G(m))|-1$, then $i>|V(G(m))|$, and by Lemma 1, $\mathscr{D}(G(m), i)=\emptyset$, a contradiction. So we have $i-1<\gamma(G(m-2))$, and since $\mathscr{D}(G(m-3), i-1) \neq \emptyset$, together we have $\gamma(G(m-3)) \leq i-1<\gamma(G(m-2))$. So $\gamma(G(m-3))+1 \leq$ $i<\gamma(G(m-2))+1$.

$(\Leftarrow)$ If $\gamma(G(m-3))+1 \leq i<\gamma(G(m-2))+1$, then by Lemma 1, we have $\mathscr{D}(G(m-1), i-1)=\mathscr{D}(G(m-2), i-1)=\emptyset$ and $\mathscr{D}(G(m-3), i-1) \neq \emptyset$.

(ii) $(\Rightarrow)$ Since $\mathscr{D}(G(m-2), i-1)=\mathscr{D}(G(m-3), i-1)=\emptyset$, by Lemmas 1 and 3, $i-1>|V(G(m))|-2$ or $i-1<\gamma(G(m-3))$. If $i-1<\gamma(G(m-3))$, then $i-1<\gamma(G(m-1))$, and hence $\mathscr{D}(G(m-1), i-1)=\emptyset$, a contradiction. So we must have $i-1>|V(G(m))|-2$. Also since $\mathscr{D}(G(m-1), i-1) \neq \emptyset$, we have $i-1 \leq|V(G(m))|-1$. Therefore we have $i=|V(G(m))|$.

$(\Leftarrow)$ If $i=|V(G(m))|$, then by Lemma 1, we have $\mathscr{D}(G(m-$ $2), i-1)=\mathscr{D}(G(m-3), i-1)=\emptyset$ and $\mathscr{D}(G(m-1), i-1) \neq \emptyset$.

(iii) $(\Rightarrow)$ Since $\mathscr{D}(G(m-1), i-1)=\emptyset$, by Lemma 1 , $i-1>|V(G(m))|-1$ or $i-1<\gamma(G(m-1))$. If $i-1>$ $|V(G(m))|-1$, then $i-1>|V(G(m))|-2$, and by Lemma 1 , 
$\mathscr{D}(G(m-2), i-1)=\mathscr{D}(G(m-3), i-1)=\emptyset$, a contradiction. So we must have $i-1<\gamma(G(m-1))$. But we also have $i-1 \geq \gamma(G(m-2))$ because $\mathscr{D}(G(m-2), i-1) \neq \emptyset$. Hence, we have $\gamma(G(m-2))+1 \leq i<\gamma(G(m-1))+1$.

$(\Leftarrow)$ If $\gamma(G(m-2))+1 \leq i<\gamma(G(m-1))+1$, then by Lemma $1, \mathscr{D}(G(m-1), i-1)=\emptyset, \mathscr{D}(G(m-2), i-1) \neq \emptyset$ and $\mathscr{D}(G(m-3), i-1) \neq \emptyset$.

(iv) $(\Rightarrow)$ Since $\mathscr{D}(G(m-3), i-1)=\emptyset$, by Lemma 1 , we have $i-1>|V(G(m))|-3$ or $i-1<\gamma(G(m-3))$. Since $\mathscr{D}(G(m-2)), i-1) \neq \emptyset$, by Lemma 1 , we have $\gamma(G(m-2)) \leq$ $i-1 \leq|V(G(m))|-2$. Therefore $i-1<\gamma(G(m-3))$ is not possible. Hence we must have $i-1>|V(G(m))|-3$. Thus $i=|V(G(m))|-1$ or $|V(G(m))|$. But $i \neq|V(G(m))|$ because $\mathscr{D}(G(m-3), i-1)=\emptyset$. So we have $i=|V(G(m))|-1$.

$(\Leftarrow)$ If $i=|V(G(m))|-1$, then by Lemma $1, \mathscr{D}(G(m-$ $1), i-1) \neq \emptyset, \mathscr{D}(G(m-2), i-1) \neq \emptyset$ and $\mathscr{D}(G(m-3), i-1)=\emptyset$.

$(\mathrm{v})(\Rightarrow)$ Since $\mathscr{D}(G(m-1), i-1) \neq \emptyset, \mathscr{D}(G(m-2), i-1) \neq \emptyset$, and $\mathscr{D}(G(m-3), i-1) \neq \emptyset$, then by applying Lemma 1 , we have $\gamma(G(m-1)) \leq i-1 \leq|V(G(m))|-1, \gamma(G(m-2)) \leq i-1 \leq$ $|V(G(m))|-2$, and $\gamma(G(m-3)) \leq i-1 \leq|V(G(m))|-3$. So, by Lemma 3, $\gamma(G(m-1)) \leq i-1 \leq|V(G(m))|-3$, and hence $\gamma(G(m-1))+1 \leq i \leq|V(G(m))|-2$.

$(\Leftarrow)$ If $\gamma(G(m-1))+1 \leq i \leq|V(G(m))|-2$, then by Lemma 1 we have the result.

By Lemma 6, for the construction of $\mathscr{D}(G(m), i)$, suffices to consider $\mathscr{D}(G(m-1), i-1), \mathscr{D}(G(m-2), i-1)$, and $\mathscr{D}(G(m-$ $3), i-1)$. By Lemma 7 , we need only to consider the five cases in the following theorem.

\section{Theorem 9. For every $i \geq \gamma(G(m))$,}

(i) if $\mathscr{D}(G(m-1), i-1)=\mathscr{D}(G(m-2), i-1)=\emptyset$ and $\mathscr{D}(G(m-3), i-1) \neq \emptyset$, then $\mathscr{D}(G(m), i)=\left\{\left\{y_{m-1}\right\} \cup X \mid\right.$ $X \in \mathscr{D}(G(m-3), i-1)\}$;

(ii) if $\mathscr{D}(G(m-2), i-1)=\mathscr{D}(G(m-3), i-1)=$ $\emptyset$ and $\mathscr{D}(G(m-1), i-1) \neq \emptyset$, then $\mathscr{D}(G(m), i)=$ $\mathscr{D}(G(m),|V(G(m))|)=\left\{\left\{y_{m}\right\} \cup X \mid X \in \mathscr{D}(G(m-\right.$ $1), i-1)\}$;

(iii) if $\mathscr{D}(G(m-1), i-1)=\emptyset, \mathscr{D}(G(m-2), i-1) \neq \emptyset$ and $\mathscr{D}(G(m-3), i-1) \neq \emptyset$, then $\mathscr{D}(G(m), i)=\left\{\left\{y_{m}\right\} \cup\right.$ $X_{1},\left\{y_{m-1}\right\} \cup X_{2} \mid X_{1} \in \mathscr{D}(G(m-2), i-1), X_{2} \in$ $\mathscr{D}(G(m-3), i-1)\}$;

(iv) if $\mathscr{D}(G(m-1), i-1) \neq \emptyset, \mathscr{D}(G(m-2), i-1) \neq \emptyset$ and $\mathscr{D}(G(m-3), i-1)=\emptyset$, then $\mathscr{D}(G(m), i)=\left\{\left\{y_{m}\right\} \cup\right.$ $X_{1},\left\{y_{m-1}\right\} \cup X_{2} \mid X_{1} \in \mathscr{D}(G(m-1), i-1), X_{2} \in$ $\mathscr{D}(G(m-2), i-1)\}$;

(v) if $\mathscr{D}(G(m-1), i-1) \neq \emptyset, \mathscr{D}(G(m-2), i-1) \neq \emptyset$ and $\mathscr{D}(G(m-3), i-1) \neq \emptyset$ are nonempty, then $\mathscr{D}(G(m), i)=$ $\left\{\left\{y_{m}\right\} \cup X_{1},\left\{y_{m-1}\right\} \cup X_{2} \mid X_{1} \in \mathscr{D}(G(m-1), i-1), X_{2} \in\right.$ $\mathscr{D}(G(m-2), i-1)\} \cup\left\{\left\{y_{m-1}\right\} \cup X \mid X \in \mathscr{D}(G(m-3), i-\right.$ 1) $\backslash \mathscr{D}(G(m-2), i-1)\} \cup\left\{\left\{y_{m}\right\} \cup X \mid X \in \mathscr{D}(G(m-3)\right.$, $i-1) \cap \mathscr{D}(G(m-2), i-1)\}$.

Proof. (i) Obviously $\left\{\left\{y_{m-1}\right\} \cup X \mid X \in \mathscr{D}(G(m-3), i-\right.$ $1)\} \subseteq \mathscr{D}(G(m), i)$. Now suppose that $Y \in \mathscr{D}(G(m), i)$, then at least one of the vertices $y_{m}$ or $y_{m-1}$ is in $Y$. If $y_{m} \in Y$ then by Lemma 5 at least one of the vertices $y_{m-1}, y_{m-2}$, or $y_{m-3}$ is in $Y$. If $y_{m-1} \in Y$ or $y_{m-2} \in Y$, then $Y-\left\{y_{m}\right\} \in$ $\mathscr{D}(G(m-1), i-1)$, a contradiction. If $y_{m-3} \in Y$, then $Y-\left\{y_{m}\right\} \in \mathscr{D}(G(m-2), i-1)$, a contradiction. Now suppose that $y_{m-1} \in Y$, then by Lemma 5 at least one of the vertices $y_{m-2}, y_{m-3}$, or $y_{m-4}$ is in $Y$. If $y_{m-2} \in Y$ or $y_{m-3} \in Y$, then $Y-\left\{y_{m-1}\right\} \in \mathscr{D}(G(m-2), i-1)$, a contradiction. If $y_{m-4} \in Y$, then $Y-\left\{y_{m-1}\right\} \in \mathscr{D}(G(m-3), i-1)$. So $\mathscr{D}(G(m), i) \subseteq$ $\left\{\left\{y_{m-1}\right\} \cup X \mid X \in \mathscr{D}(G(m-3), i-1)\right\}$.

(ii) By Lemma 8(ii), $i=|V(G(m))|$. If we suppose that $G(m)$ has labeled with numbers in $[|V(G(m))|]$, then $\mathscr{D}(G(m), i)=\mathscr{D}(G(m),|V(G)|)=\{[|V(G)|]\}=\left\{\left\{y_{m}\right\} \cup X \mid\right.$ $X \in \mathscr{D}(G(m-1), i-1)\}$.

(iii) It is obvious that $\left\{\left\{y_{m}\right\} \cup X_{1},\left\{y_{m-1}\right\} \cup X_{2} \mid X_{1} \in\right.$ $\left.\mathscr{D}(G(m-2), i-1), X_{2} \in \mathscr{D}(G(m-3), i-1)\right\} \subseteq \mathscr{D}(G(m), i)$. Now let $Y \in \mathscr{D}(G(m), i)$. Then $y_{m}$ or $y_{m-1}$ is in $Y$. If $y_{m} \in Y$, then by Lemma 5 , at least one vertices $y_{m-1}, y_{m-2}$, or $y_{m-3}$ is in $Y$. If $y_{m-1}$ or $y_{m-2}$ is in $Y$, then $Y-\left\{y_{m}\right\} \in \mathscr{D}(G(m-$ $1), i-1)$, a contradiction, because $\mathscr{D}(G(m-1), i-1)=\emptyset$. Hence, $y_{m-3} \in Y, y_{m-2} \notin Y$, and $y_{m-1} \notin Y$. Therefore $Y=$ $X \cup\left\{y_{m}\right\}$ for some $X \in \mathscr{D}(G(m-2), i-1)$. Now suppose that $y_{m-1} \in Y$ and $y_{m} \notin Y$. By Lemma 5, at least one vertex labeled $y_{m-2}, y_{m-3}$, or $y_{m-4}$ is in $Y$. By Lemma 8(iii), $\gamma(G(m-$ $2)) \leq i-1<\gamma(G(m-1)) \leq \gamma(G(m))$, and on the other hand since $\mathscr{D}(G(m), i) \neq \emptyset$, then $i \geq \gamma(G(m))$. So together we have $i=\gamma(G(m))$. Therefore $\mathscr{D}(G(m), i)=\Gamma(G(m))$, and so all these sets in $\Gamma(G(m))$ have minimum cardinality. Therefore, since $Y \in \mathscr{D}(G(m), i)$ and $y_{m-1} \in Y$, then $y_{m-2} \notin Y$. Hence $y_{m-3}$ or $y_{m-4}$ is in $Y$, but $y_{m-2} \notin Y$. Thus $Y=X \cup\left\{y_{m-1}\right\}$ for some $X \in \mathscr{D}(G(m-3), i-1)$. So $\mathscr{D}(G(m), i) \subseteq\left\{\left\{y_{m}\right\} \cup X_{1}\right.$, $\left.\left\{y_{m-1}\right\} \cup X_{2} \mid X_{1} \in \mathscr{D}(G(m-2), i-1), X_{2} \in \mathscr{D}(G(m-3), i-1)\right\}$.

(iv) By Lemma 8, $i=|V(G(m))|-1$. If we suppose that $G(m)$ has labeled with numbers in $[|V(G(m))|]$, then $\mathscr{D}(G(m), i)=\{[|V(G(m))|]-\{x\} \mid x \in[|V(G(m))|]\}=$ $\left\{\left\{y_{m}\right\} \cup X_{1},\left\{y_{m-1}\right\} \cup X_{2} \mid X_{1} \in \mathscr{D}(G(m-1), i-1)\right.$, $\left.X_{2} \in \mathscr{D}(G(m-2), i-1)\right\}$.

(v) $\mathscr{D}(G(m-1), i-1) \neq \emptyset, \mathscr{D}(G(m-2), i-1) \neq \emptyset$ and $\mathscr{D}(G(m-3), i-1) \neq \emptyset$. Let $X \in \mathscr{D}(G(m-1), i-1)$, so at least one vertex labeled $y_{m-1}$ or $y_{m-2}$ is in $X$. If $y_{m-1}$ or $y_{m-2} \in X$, then $X \cup\left\{y_{m}\right\} \in \mathscr{D}(G(m), i)$. Now let $X \in \mathscr{D}(G(m-2), i-1)$, then $y_{m-2}$ or $y_{m-3}$ is in $X$. If $y_{m-2}$ or $y_{m-3} \in X$, then $X \cup\left\{y_{m-1}\right\} \in$ $\mathscr{D}(G(m), i)$. Now let $X \in \mathscr{D}(G(m-3), i-1)$, then $y_{m-3}$ or $y_{m-4}$ is in $X$. If $y_{m-3} \in X$, then $X \cup\{x\} \in \mathscr{D}(G(m), i)$, for $x \in\left\{y_{m}, y_{m-1}\right\}$. If $y_{m-4} \in X$, then $X \cup\left\{y_{m-1}\right\} \in \mathscr{D}(G(m), i)$. Therefore we have $\left\{\left\{y_{m}\right\} \cup X_{1},\left\{y_{m-1}\right\} \cup X_{2} \mid X_{1} \in \mathscr{D}(G(m-\right.$ $\left.1), i-1), X_{2} \in \mathscr{D}(G(m-2), i-1)\right\} \cup\left\{\left\{y_{m-1}\right\} \cup X \mid X \in\right.$ $\mathscr{D}(G(m-3), i-1) \backslash \mathscr{D}(G(m-2), i-1)\} \cup\left\{\left\{y_{m}\right\} \cup X \mid X \in\right.$ $\mathscr{D}(G(m-3), i-1) \cap \mathscr{D}(G(m-2), i-1)\} \subseteq \mathscr{D}(G(m), i)$. Now, let $Y \in \mathscr{D}(G(m), i)$, then $y_{m} \in Y$ or $y_{m-1} \in Y$. If $y_{m} \in Y$, then by Lemma 5, at least one vertex labeled $y_{m-1}, y_{m-2}$, or $y_{m-3}$ is in $Y$. If $y_{m-1} \in Y$ or $y_{m-2} \in Y$, then $Y=X \cup\left\{y_{m}\right\}$ for some $X \in \mathscr{D}(G(m-1), i-1)$. If $y_{m-3} \in Y, y_{m-2} \notin Y$, and $y_{m-1} \notin Y$, then $Y=X \cup\left\{y_{m}\right\}$ for some $X \in \mathscr{D}(G(m-2), i-1) \cap \mathscr{D}(G(m-3), i-1)$. Now suppose that $y_{m-1} \in Y$ and $y_{m} \notin Y$, then by Lemma 5 , at least one vertex labeled $y_{m-2}, y_{m-3}$, or $y_{m-4}$ is in $Y$. If $y_{m-2} \in Y$ or $y_{m-3} \in Y$, then $Y=X \cup\left\{y_{m-1}\right\}$ for some $X \in \mathscr{D}(G(m-2), i-1)$. If $y_{m-4} \in Y, y_{m-3} \notin Y$, and $y_{m-2} \notin Y$, then $Y=X \cup\left\{y_{m-1}\right\}$ for some $X \in \mathscr{D}(G(m-3), i-1) \backslash \mathscr{D}(G(m-2), i-1)$. So $\mathscr{D}(G(m), i) \subseteq\left\{\left\{y_{m}\right\} \cup X_{1},\left\{y_{m-1}\right\} \cup X_{2} \mid X_{1} \in \mathscr{D}(G(m-\right.$ 
$\left.1), i-1), X_{2} \in \mathscr{D}(G(m-2), i-1)\right\} \cup\left\{\left\{y_{m-1}\right\} \cup X \mid X \in\right.$ $\mathscr{D}(G(m-3), i-1) \backslash \mathscr{D}(G(m-2), i-1)\} \cup\left\{\left\{y_{m}\right\} \cup X \mid X \in\right.$ $\mathscr{D}(G(m-3), i-1) \cap \mathscr{D}(G(m-2), i-1)\}$.

Theorem 10. For any $m \geq 3$ and $i \geq \gamma(G(m))$,

$$
\begin{aligned}
|\mathscr{D}(G(m), i)|= & |\mathscr{D}(G(m-1), i-1)|+|\mathscr{D}(G(m-2), i-1)| \\
& +|\mathscr{D}(G(m-3), i-1)| .
\end{aligned}
$$

Proof. It follows from Theorem 9.

Theorem 11. For any $m \geq 3$,

$$
\begin{gathered}
D(G(m), x)=x(D(G(m-1), x)+D(G(m-2), x) \\
+D(G(m-3), x)) .
\end{gathered}
$$

Proof. We have the result by definition of domination polynomial and Theorem 10.

\section{Dominating Sets of Paths and Some Other Graphs}

First we investigate the dominating sets of paths. Since $P_{n}=$ $P_{1}(n-1)$, we use the results for graph $G(m)$ in the previous section to obtain properties of dominating sets of path. We suppose that $P_{n}$ is labeled as in Figure 1(a) and denote $\mathscr{D}\left(P_{n}, i\right)$ simply by $P_{i}^{n}$.

Lemma 12. Suppose that $\mathscr{P}_{i}^{n} \neq \emptyset$, then one has

(i) $\mathscr{P}_{i-1}^{n-1}=\mathscr{P}_{i-1}^{n-2}=\emptyset$ and $\mathscr{P}_{i-1}^{n-3} \neq \emptyset$ if and only if $n=3 k$ and $i=k$ for some $k \in N$,

(ii) $\mathscr{P}_{i-1}^{n-2}=\mathscr{P}_{i-1}^{n-3}=\emptyset$ and $\mathscr{P}_{i-1}^{n-1} \neq \emptyset$ if and only if $i=n$,

(iii) $\mathscr{P}_{i-1}^{n-1}=\emptyset, \mathscr{P}_{i-1}^{n-2} \neq \emptyset$, and $\mathscr{P}_{i-1}^{n-3} \neq \emptyset$ if and only if $n=$ $3 k+2$ and $i=\lceil(3 k+2) / 3\rceil$ for some $k \in N$,

(iv) $\mathscr{P}_{i-1}^{n-1} \neq \emptyset, \mathscr{P}_{i-1}^{n-2} \neq \emptyset$, and $\mathscr{P}_{i-1}^{n-3}=\emptyset$ if and only if $i=$ $n-1$,

(v) $\mathscr{P}_{i-1}^{n-1} \neq \emptyset, \mathscr{P}_{i-1}^{n-2} \neq \emptyset$, and $\mathscr{P}_{i-1}^{n-3} \neq \emptyset$ if and only if $\lceil(n-$ 1) $/ 3\rceil+1 \leq i \leq n-2$.

Proof. (i) $(\Rightarrow)$ By Lemmas 4 and 8(i), we have $\lceil n / 3\rceil \leq i<$ $\lceil(n-2) / 3\rceil+1$, which give us $n=3 k$ and $i=k$ for some $k \in N$.

$(\Leftarrow)$ It follows from Lemmas 4 and $8(\mathrm{i})$.

(ii) It follows from Lemma 8(ii).

(iii) $(\Rightarrow)$ By Lemmas 4 and 8 (iii), we have $\lceil(n-2) / 3\rceil+1 \leq$ $i<\lceil(n-1) / 3\rceil+1$, which give us $n=3 k+2$ and $i=k+1=$ $\lceil(3 k+2) / 3\rceil$ for some $k \in N$.

$(\Leftarrow)$ It follows from Lemmas 4 and 8 (iii).

(iv) It follows from Lemma 8 (iv).

(v) It follows from Lemmas 4 and $8(\mathrm{v})$.

The following theorem specify $\mathscr{P}_{i}^{n}$.

Theorem 13. For every $n \geq 4$ and $i \geq\lceil n / 3\rceil$,

$$
\mathscr{P}_{i}^{n}= \begin{cases}\{\{2,5, \ldots, n-4, n-1\}\}, & \text { if } \mathscr{P}_{i-1}^{n-1}=\mathscr{P}_{i-1}^{n-2}=\emptyset, \\ \{[n]\}, & \text { if } \mathscr{P}_{i-1}^{n-2}=\mathscr{P}_{i-1}^{n-3}=\emptyset, \\ \{\{n]-\{x\} \mid x \in[n]\}, & \text { if } \mathscr{P}_{i-1}^{n-1}=\emptyset, \\ \left\{\{n\} \cup X_{1},\{n-1\} \cup X_{2} \mid X_{1} \in \mathscr{P}_{i-1}^{n-1}, X_{2} \in \mathscr{P}_{i-1}^{n-2}\right\} & \text { if } \mathscr{P}_{i-1}^{n-3}=\emptyset, \\ \cup\left\{\{n-1\} \cup X \mid X \in \mathscr{P}_{i-1}^{n-3} \backslash \mathscr{P}_{i-1}^{n-2}\right\} & \\ \cup\left\{\{n\} \cup X \mid X \in \mathscr{P}_{i-1}^{n-3} \cap \mathscr{P}_{i-1}^{n-2}\right\}, & \text { if } \mathscr{P}_{i-1}^{n-1} \neq \emptyset, \mathscr{P}_{i-1}^{n-2} \neq \emptyset, \mathscr{P}_{i-1}^{n-3} \neq \emptyset .\end{cases}
$$

Proof.

Case 1. $\mathscr{P}_{i-1}^{n-1}=\mathscr{P}_{i-1}^{n-2}=\emptyset$ and $\mathscr{P}_{i-1}^{n-3} \neq \emptyset$. By Lemma 12(i), $n=3 k$ and $i=k$ for some $k \in N$. Therefore $\mathscr{P}_{i}^{n}=\mathscr{P}_{n / 3}^{n}=$ $\{\{2,5, \ldots, n-4, n-1\}\}$.

Case 2. It follows from Theorem 9(ii).

Case 3. By Theorem 9(iii), $\mathscr{P}_{i}^{n}=\left\{\{n\} \cup X_{1},\{n-1\} \cup X_{2} \mid X_{1} \in\right.$ $\left.\mathscr{P}_{i-1}^{n-2}, X_{2} \in \mathscr{P}_{i-1}^{n-3}\right\}$. By Lemma 12(iii), $n=3 k+2$ and $i=k+1$ for some $k \in N$. Since $\mathscr{P}_{i-1}^{n-2}=\mathscr{P}_{k}^{3 k}=\{\{2,5, \ldots, 3 k-1\}\}$, then $\left\{X \cup\{3 k+2\} \mid X \in \mathscr{P}_{k}^{3 k}\right\}=\{\{2,5, \ldots, 3 k-1,3 k+2\}\}$. Therefore $\mathscr{P}_{i}^{n}=\{\{2,5, \ldots, n-3, n\}\} \cup\left\{X \cup\{n-1\} \mid X \in \mathscr{P}_{i-1}^{n-3}\right\}$.

Cases 4 and 5 follow from Theorem 9(iv) and (v), respectively.

Example 14. We use Theorem 13 to construct $\mathscr{P}_{i}^{6}$ for $2 \leq i \leq 6$. Since $\mathscr{P}_{1}^{5}=\mathscr{P}_{1}^{4}=\emptyset$ and $\mathscr{P}_{1}^{3}=\{\{2\}\}$, by Theorem 13, $\mathscr{P}_{2}^{6}=\{\{2,5\}\}$. Since $\mathscr{P}_{5}^{5}=\{[5]\}, \mathscr{P}_{5}^{4}=\emptyset$, and $\mathscr{P}_{5}^{3}=\emptyset$, 


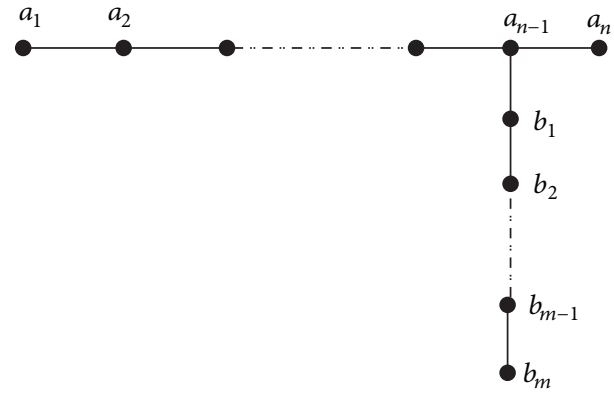

FIgURE 2: The tree $T_{n}(m)$.

we get $\mathscr{P}_{6}^{6}=\{[6]\}$. Since $\mathscr{P}_{4}^{5}=\{\{1,2,3,4\},\{1,2,3,5\}$, $\{1,3,4,5\},\{2,3,4,5\}, \quad\{1,2,4,5\}\}, \mathscr{P}_{4}^{4}=\{[4]\}$, and $\mathscr{P}_{4}^{3}=\emptyset$, then by Theorem 13, $\mathscr{P}_{5}^{6}=\{[6]-\{x\} \mid$ $x \in[6]\}=\{\{1,2,3,4,6\},\{1,2,3,5,6\},\{1,3,4,5,6\}$, $\{2,3,4,5,6\},\{1,2,4,5,6\},\{1,2,3,4,5\}\}$. And, for construction $\mathscr{P}_{3}^{6}=\{\{1,3,5\},\{1,3,6\},\{2,3,6\},\{2,3,5\},\{1,4,6\},\{1,4,5\}$, $\{2,5,6\},\{2,4,6\},\{2,4,5\}, \quad\{1,2,5\}\}, \quad$ by Theorem 13, $\mathscr{P}_{3}^{6}=\left\{X_{1} \cup\{6\}, X_{2} \cup\{5\} \mid X_{1} \in \mathscr{P}_{2}^{5}, X_{2} \in \mathscr{P}_{2}^{4}\right\} \cup\{\{1,2\} \cup\{5\}$, $\{1,3\} \cup\{6\},\{2,3\} \cup\{6\}\}$. Finally, since $\mathscr{P}_{3}^{5}=\{\{1,3,5\}$, $\{1,2,4\}, \quad\{2,4,5\}, \quad\{2,3,4\}, \quad\{2,3,5\}, \quad\{1,4,5\}, \quad\{1,3,4\}$, $\{1,2,5\}\}, \mathscr{P}_{3}^{4}=\{\{1,2,3\},\{1,2,4\},\{2,3,4\},\{1,3,4\}\}$, and $\mathscr{P}_{3}^{3}=\{[3]\}$, then $\mathscr{P}_{4}^{6}=\left\{X_{1} \cup\{6\}, X_{2} \cup\{5\} \mid X_{1} \in \mathscr{P}_{3}^{5}\right.$, $\left.X_{2} \in \mathscr{P}_{3}^{4}\right\} \cup\left\{X \cup\{6\} \mid X \in \mathscr{P}_{3}^{3}\right\}=\{\{1,2,3,5\},\{1,2,3,6\}$, $\{1,2,4,6\}, \quad\{1,3,5,6\}, \quad\{1,3,4,6\}, \quad\{1,3,4,5\}, \quad\{1,2,5,6\}$, $\{1,2,4,5\}, \quad\{2,3,4,6\}, \quad\{1,4,5,6\}, \quad\{2,3,4,5\}, \quad\{2,4,5,6\}$, $\{2,3,5,6\}\}$.

Theorem 15. If $\mathscr{P}_{i}^{n}$ is the family of dominating set of $P_{n}$ with cardinality $i$, then $\left|\mathscr{P}_{i}^{n}\right|=\left|\mathscr{P}_{i-1}^{n-1}\right|+\left|\mathscr{P}_{i-1}^{n-2}\right|+\left|\mathscr{P}_{i-1}^{n-3}\right|$.

Proof. It follows from Theorem 10.

Remark 16. We have obtained [12, Lemma 12 and Theorem 5] with a different approach.

As other examples of graph $G(m)$, we study the dominating sets of some other graphs. By Theorem 10, the recursive formula in this theorem is true for every graphs which contain a gluing path with at least 3 vertices. Also, if we have the domination number of graphs, then we can study the properties of their dominating sets by Theorem 9 and Lemma 8. Here, we consider a special case of graph $P_{n}(m)$ which is a tree. We denote this tree by $T_{n}(m)$. In other words $T_{n}(m)$ is a tree, such that $T_{n}(0)=P_{n}$, and for $m \geq 1, T_{n}(m)=$ $(A, B, E)$ where $A \cup B$ is its vertex set, $A=\left\{a_{1}, \ldots, a_{n}\right\}, B=$ $\left\{b_{1}, \ldots, b_{m}\right\}$, and the edge set $E=\left\{a_{i} a_{i+1}: 1 \leq i \leq n-\right.$ $1\} \cup\left\{b_{i} b_{i+1}: 1 \leq i \leq m-1\right\} \cup\left\{a_{n-1} b_{1}\right\}$; see Figure 2 . For obtaining the domination number of $T_{n}(m)$, we need the following lemma which follows from observation.

Lemma 17. For every $n \geq 0$ and $m \geq 3, \gamma\left(T_{n}(m)\right)=$ $\gamma\left(T_{m-2}(n+2)\right)$.
Theorem 18. For every $n \geq 3$ and $m \geq 0, \gamma\left(T_{n}(m)\right)=\lceil n / 3\rceil+$ $\lceil(m-1) / 3\rceil$.

Proof. By induction on $m$. If $m=0$, then $\gamma\left(T_{n}(0)\right)=\gamma\left(P_{n}\right)=$ $\lceil n / 3\rceil$. Now suppose that the theorem is true for all numbers less than or equal $m-1$, and we prove it for $m$. By applying Theorem 2 for $e=a_{n-1} b_{1}$, we have the following inequalities:

$$
\left\lceil\frac{n}{3}\right\rceil+\left\lceil\frac{m}{3}\right\rceil-1 \leq \gamma\left(T_{n}(m)\right) \leq\left\lceil\frac{n}{3}\right\rceil+\left\lceil\frac{m}{3}\right\rceil .
$$

Similarly by applying Theorem 2 for $e=b_{2} b_{3}, b_{m-3} b_{m-2}$ and induction hypothesis, we have the following inequalities; respectively,

$$
\begin{aligned}
\left\lceil\frac{n}{3}\right\rceil+\left\lceil\frac{m-2}{3}\right\rceil & \leq \gamma\left(T_{n}(m)\right) \\
& \leq\left\lceil\frac{n}{3}\right\rceil+\left\lceil\frac{m-2}{3}\right\rceil+1, \\
\left\lceil\frac{n}{3}\right\rceil+\left\lceil\frac{m-4}{3}\right\rceil & \leq \gamma\left(T_{n}(m)\right) \\
& \leq\left\lceil\frac{n}{3}\right\rceil+\left\lceil\frac{m-4}{3}\right\rceil+1 .
\end{aligned}
$$

Now if $m=3 k$ for some $k \in N$, then by (4) and (5) we have $\gamma\left(T_{n}(m)\right)=\lceil n / 3\rceil+k=\lceil n / 3\rceil+\lceil(m-1) / 3\rceil$.

If $m=3 k+1$ for some $k \in N$, then by (4) and (6) we have $\gamma\left(T_{n}(m)\right)=\lceil n / 3\rceil+k=\lceil n / 3\rceil+\lceil(m-1) / 3\rceil$.

Now if $m=3 k+2$ for some $k \in N$, we will consider the following cases.

(i) One has $n=3 k^{\prime}$ for some $k^{\prime} \in N$. By applying Theorem 2 for $a_{n-1} a_{n}$, we have the following inequalities:

$$
\left\lceil\frac{n+m-1}{3}\right\rceil \leq \gamma\left(T_{n}(m)\right) \leq\left\lceil\frac{n+m-1}{3}\right\rceil+1 .
$$

Now, by (4) and (7), we have $\gamma\left(T_{3 k^{\prime}}(3 k+2)\right)=k+k^{\prime}+$ $1=\lceil n / 3\rceil+\lceil(m-1) / 3\rceil$.

(ii) One has $n=3 k^{\prime}+2$ for some $k^{\prime} \in N$. By Theorem 2 for $e=a_{1} a_{2}$ we have

$$
\begin{aligned}
\left\lceil\frac{n-1}{3}\right\rceil+\left\lceil\frac{m-1}{3}\right\rceil & \leq \gamma\left(T_{n}(m)\right) \\
& \leq\left\lceil\frac{n-1}{3}\right\rceil+\left\lceil\frac{m-1}{3}\right\rceil+1 .
\end{aligned}
$$

By (4) and (8), we have $\gamma\left(T_{3 k^{\prime}+2}(3 k+2)\right)=k+k^{\prime}+2=$ $\lceil n / 3\rceil+\lceil(m-1) / 3\rceil$.

(iii) One has $n=3 k^{\prime}+1$ for some $k^{\prime} \in N$. We will prove that $\gamma\left(T_{3 k^{\prime}+1}(3 k+2)\right)=k+k^{\prime}+2$. We do it by induction on $k$. If $k=0$, then by Lemma 17 , $\gamma\left(T_{3 k^{\prime}+1}(2)\right)=\gamma\left(T_{4}\left(3 k^{\prime}-1\right)\right)=2+k^{\prime}$. So the result is true for $k=0$. Now suppose that the result is true for all number less than $k$, and we prove it for $k$. 
By Lemma 17 and the induction hypothesis, we have the following equalities for $k^{\prime}-1<k$ :

$$
\begin{aligned}
\gamma\left(T_{3 k^{\prime}+1}(3 k+2)\right) \\
\quad=\gamma\left(T_{3 k+4}\left(3 k^{\prime}-1\right)\right) \\
\quad=\gamma\left(T_{3(k+1)+1}\left(3\left(k^{\prime}-1\right)+2\right)\right) \\
\quad=k^{\prime}-1+k+1+2=k^{\prime}+k+2 .
\end{aligned}
$$

If $k^{\prime}-1>k$, then for some $t>0, k^{\prime}=k+1+t$. Again by Lemma 17 ,

$$
\begin{aligned}
\gamma\left(T_{3 k^{\prime}+1}(3 k+2)\right) \\
\quad=\gamma\left(T_{3 k+3 t+4}(3 k+2)\right) \\
=\gamma\left(T_{3 k+4}(3(k+t)+2)\right) \\
=k+t+k+1+2=k^{\prime}+k+2 .
\end{aligned}
$$

Finally, for $k^{\prime}-1=k$, we have $\gamma\left(T_{3 k^{\prime}+1}(3 k+2)\right)=$ $\gamma\left(T_{3 k+4}(3 k+2)\right)=\gamma\left(T_{3(k+1)+1}(3 k+2)=2 k+3=\right.$ $k+k^{\prime}+2$. Therefore in all cases $\gamma\left(T_{n}(m)\right)=\lceil n / 3\rceil+$ $\lceil(m-1) / 3\rceil$.

Let $\mathscr{D}\left(T_{n}(m), i\right)$ or simply $\mathscr{D}_{i}^{n, m}$ be the family of dominating set of $T_{n}(m)$ with cardinality $i$. By Lemma 8 and Theorem 9 we prove the following lemma, which is some properties of $\mathscr{D}_{i}^{n, m}$.

Lemma 19. Suppose that $\mathscr{D}_{i}^{n, m} \neq \emptyset$, then

(i) $\mathscr{D}_{i-1}^{n, m-1}=\mathscr{D}_{i-1}^{n, m-2}=\emptyset$ and $\mathscr{D}_{i-1}^{n, m-3} \neq \emptyset$ if and only if $m=3 k+1, i=\lceil n / 3\rceil+k$, for some $k \in N$,

(ii) $\mathscr{D}_{i-1}^{n, m-2}=\mathscr{D}_{i-1}^{n, m-3}=\emptyset$, and $\mathscr{D}_{i-1}^{n, m-1} \neq \emptyset$ if and only if $i=n+m$,

(iii) $\mathscr{D}_{i-1}^{n, m-1}=\emptyset, \mathscr{D}_{i-1}^{n, m-2} \neq \emptyset$, and $\mathscr{D}_{i-1}^{n, m-3} \neq \emptyset$ if and only if $m=3 k, i=\lceil n / 3\rceil+k$, for some $k \in N$,

(iv) $\mathscr{D}_{i-1}^{n, m-1} \neq \emptyset, \mathscr{D}_{i-1}^{n, m-2} \neq \emptyset$, and $\mathscr{D}_{i-1}^{n, m-3}=\emptyset$, if and only if $i=m+n-1$,

(v) $\mathscr{D}_{i-1}^{n, m-1} \neq \emptyset, \mathscr{D}_{i-1}^{n, m-2} \neq \emptyset$ and $\mathscr{D}_{i-1}^{n, m-3} \neq \emptyset$ if and only if $\lceil n / 3\rceil+\lceil(m-2) / 3\rceil+1 \leq i \leq m+n-2$.

Proof. (i) By Lemma 8(i) and Theorem 18, $\lceil n / 3\rceil+\lceil(m-4) / 3\rceil+$ $1 \leq i<\lceil n / 3\rceil+\lceil(m-3) / 3\rceil+1$, which give us $m=3 k+1, i=$ $\lceil n / 3\rceil+k$, for some $k \in N$.

(ii) By Lemma 8(ii), $i=\left|V\left(T_{n}(m)\right)\right|=m+n$.

(iii) By Lemma 8(iii) and Theorem $18,\lceil n / 3\rceil+\lceil(m-3) / 3\rceil+$ $1 \leq i<\lceil n / 3\rceil+\lceil(m-2) / 3\rceil+1$. Therefore $m=3 k$ and $i=\lceil n / 3\rceil+k$ for some $k \in N$.

(iv) By Lemma 8(iv), $i=\left|V\left(T_{n}(m)\right)\right|-1=n+m-1$.

(v) By Lemma 8(v) and Theorem 18, $\lceil n / 3\rceil+\lceil(m-2) / 3\rceil+$ $1 \leq i \leq m+n-2$.

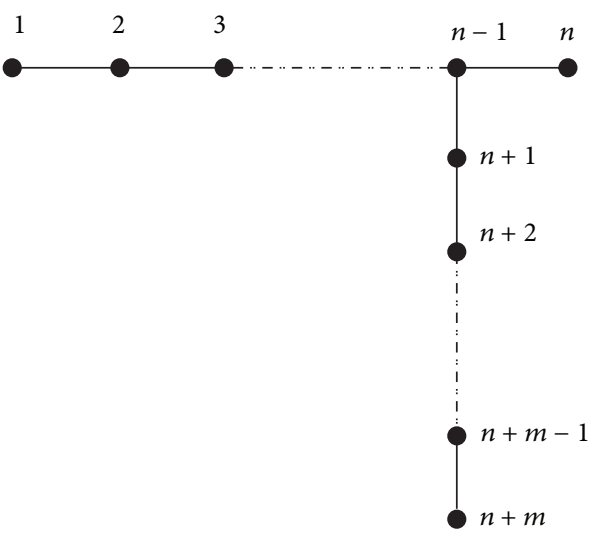

Figure 3: Labeled $T_{n}(m)$.

Here, we suppose that the tree $T_{n}(m)$ labeled as shown in Figure 3. The following theorem constructs $\mathscr{D}_{i}^{n, m}$ from $\mathscr{D}_{i-1}^{n, m-1}, \mathscr{D}_{i-1}^{n, m-2}$, and $\mathscr{D}_{i-1}^{n, m-3}$.

Theorem 20. (i) If $\mathscr{D}_{i-1}^{n, m-1}=\mathscr{D}_{i-1}^{n, m-2}=\emptyset$ and $\mathscr{D}_{i-1}^{n, m-3} \neq \emptyset$, then

$$
\mathscr{D}_{i}^{n, m}=\left\{\{m+n-1\} \cup X \mid X \in \mathscr{D}_{i-1}^{n, m-3}\right\} .
$$

(ii) If $\mathscr{D}_{i-1}^{n, m-2}=\mathscr{D}_{i-1}^{n, m-3}=\emptyset$ and $\mathscr{D}_{i-1}^{n, m-1} \neq \emptyset$, then

$$
\mathscr{D}_{i}^{n}=\left\{\{m+n\} \cup X \mid X \in \mathscr{D}_{i-1}^{n, m-1}\right\}=\{[m+n]\} .
$$

(iii) If $\mathscr{D}_{i-1}^{n, m-1}=\emptyset, \mathscr{D}_{i-1}^{n, m-2} \neq \emptyset$, and $\mathscr{D}_{i-1}^{n, m-3} \neq \emptyset$, then $\mathscr{D}_{i}^{n, m}=\left\{\{m+n\} \cup X_{1}\right.$,

$$
\left.\{m+n-1\} \cup X_{2} \mid X_{1} \in \mathscr{D}_{i-1}^{n, m-2}, X_{2} \in \mathscr{D}_{i-1}^{n, m-3}\right\} .
$$

(iv) If $\mathscr{D}_{i-1}^{n, m-3}=\emptyset, \mathscr{D}_{i-1}^{n, m-2} \neq \emptyset$, and $\mathscr{D}_{i-1}^{n, m-1} \neq \emptyset$, then

$$
\begin{aligned}
\mathscr{D}_{i}^{n}=\{ & \{m+n\} \cup X_{1}, \\
& \left.\{m+n-1\} \cup X_{2} \mid X_{1} \in \mathscr{D}_{i-1}^{n, m-1}, X_{2} \in \mathscr{D}_{i-1}^{n, m-2}\right\} .
\end{aligned}
$$

(v) If $\mathscr{D}_{i-1}^{n, m-1}, \mathscr{D}_{i-1}^{n, m-2}$, and $\mathscr{D}_{i-1}^{n, m-3}$ are nonempty, then

$$
\begin{aligned}
\mathscr{D}_{i}^{n, m}= & \left\{\{m+n\} \cup X_{1},\{m+n-1\} \cup X_{2} \mid\right. \\
& \left.X_{1} \in \mathscr{D}_{i-1}^{n, m-1}, X_{2} \in \mathscr{D}_{i-1}^{n, m-2}\right\} \\
\cup & \left\{\{m+n-1\} \cup X \mid X \in \mathscr{D}_{i-1}^{n, m-3} \backslash \mathscr{D}_{i-1}^{n, m-2}\right\} \\
\cup & \left\{\{m+n\} \cup X \mid X \in \mathscr{D}_{i-1}^{n, m-3} \cap \mathscr{D}_{i-1}^{n, m-2}\right\} .
\end{aligned}
$$

Proof. It follows from Theorem 9.

Now we obtain the following theorem from Theorem 20 or Theorem 10. 


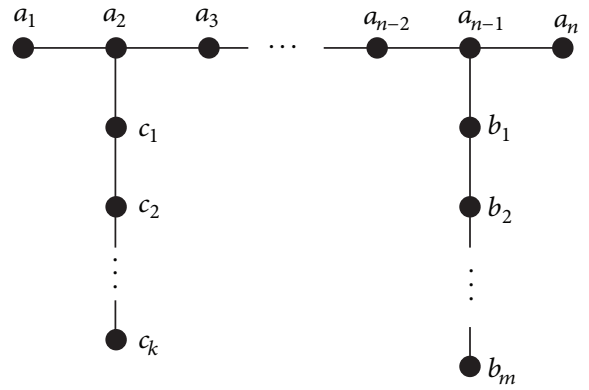

(a)

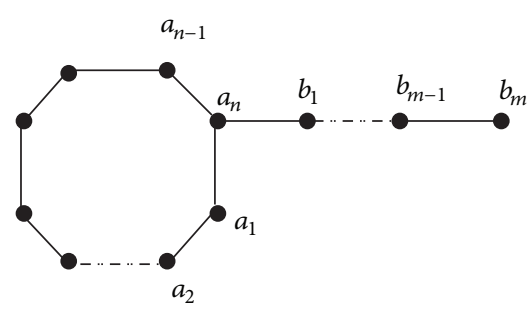

(b)

Figure 4: The graphs $T_{m, n}(k)$ and $C_{n}(m)$, respectively.

Theorem 21. If $\mathscr{D}_{i}^{n, m}$ is the family of dominating set of $T_{n}(m)$ with cardinality i, then $\left|\mathscr{D}_{i}^{n, m}\right|=\left|\mathscr{D}_{i-1}^{n, m-1}\right|+\left|\mathscr{D}_{i-1}^{n, m-2}\right|+\left|\mathscr{D}_{i-1}^{n, m-3}\right|$.

Note that the tree $T_{n}(m)$ is obtained by gluing an end vertex of a path $P_{m}$ to the vertex $a_{n-1}$ of $P_{n}$. We now consider another type of related tree $T_{m, n}(k)$ (or special case of $\left.\left(T_{n}(m)\right)(k)\right)$, which is obtained from $T_{n}(m)$ by gluing an end vertex of a path $P_{k}$ to the vertex $a_{2}$ of $P_{n}$, see Figure 4 . In other words, let $T_{m, n}(k)=(A, B, C, E), k, n \geq 0$, where $A \cup B \cup C$ is its vertex set, $A=\left\{a_{1}, \ldots, a_{n}\right\}, B=\left\{b_{1}, \ldots, b_{m}\right\}$, $C=\left\{c_{1}, \ldots, c_{k}\right\}$, and the edge set $E=\left\{a_{i} a_{i+1}: 1 \leq i \leq n-1\right\} \cup$ $\left\{b_{i} b_{i+1}: 1 \leq i \leq m-1\right\} \cup\left\{c_{i} c_{i+1}: 1 \leq i \leq k-1\right\} \cup\left\{a_{n-1} b_{1}, a_{2} c_{1}\right\}$.

Theorem 22. For every $n \geq 3$ and $m, k \geq 0, \gamma\left(T_{m, n}(k)\right)=$ $\lceil n / 3\rceil+\lceil(m-1) / 3\rceil+\lceil(k-1) / 3\rceil$.

Proof. Similar to the proof of Theorem 18.

By Theorem 10, we have the following theorem.

Theorem 23. If $\mathscr{D}_{i}^{n, m, k}$ is the family of dominating set of $T_{m, n}(k)$ with cardinality $i$, then $\left|\mathscr{D}_{i}^{n, m, k}\right|=$ $\left|\mathscr{D}_{i-1}^{n, m, k-1}\right|+\left|\mathscr{D}_{i-1}^{n, m, k-2}\right|+\left|\mathscr{D}_{i-1}^{n, m, k-3}\right|$.

As other examples for graphs $G(m)$, we consider graph $C_{n}(m)$ which obtain by gluing a path to one vertex of cycle as shown in Figure 4. By Theorem 10 we have the following theorem for $C_{n}(m)$.

Theorem 24. For everyn, $m \geq 3,\left|\mathscr{D}\left(C_{n}(m), i\right)\right|=\mid \mathscr{D}\left(C_{n}(m-\right.$ $1), i-1)|+| \mathscr{D}\left(C_{n}(m-2), i-1\right)|+| \mathscr{D}\left(C_{n}(m-3), i-1\right) \mid$.

Let sun be a graph which is obtained by gluing end vertices of paths $P_{i_{1}}, P_{i_{2}}, \ldots, P_{i_{n}}$ to the $n$ vertices of $C_{n}$.
We denote this graph by $S\left(i_{1}, i_{2}, \ldots, i_{n}\right)$. Since sun is the special case of $\left(\cdots\left(\left(C_{n}\left(i_{1}\right)\right)\left(i_{2}\right)\right) \cdots\left(i_{n}\right)\right.$, we have the following theorem for sun.

Theorem 25. Suppose that for every $1 \leq j \leq n$, $i_{j} \geq$ 3 and $i \geq \gamma\left(S\left(i_{1}, i_{2}, \ldots, i_{n}\right)\right)$, then $\left|\mathscr{D}\left(S\left(i_{1}, i_{2}, \ldots, i_{n}\right), i\right)\right|=$ $\left|\mathscr{D}\left(S\left(i_{1}, i_{2}, \ldots, i_{n}-1\right), i-1\right)\right|+\mid \mathscr{D}\left(S\left(i_{1}, i_{2}, \ldots, i_{n}-2\right), i-\right.$ $1)|+| \mathscr{D}\left(S\left(i_{1}, i_{2}, \ldots, i_{n}-3\right), i-1\right) \mid$.

\section{Acknowledgment}

The authors would like to express their gratitude to the referees for their careful reading and helpful comments.

\section{References}

[1] T. W. Haynes, S. T. Hedetniemi, and P. J. Slater, Fundamentals of Domination in Graphs, Marcel Dekker, New York, NY, USA, 1998.

[2] S. Alikhani, Dominating Sets and Domination Polynomials of Graphs [Ph.D. thesis], Universiti Putra Malaysia, 2009.

[3] S. Akbari, S. Alikhani, M. R. Oboudi, and Y. H. Peng, "On the zeros of domination polynomial of a graph," Contemporary Mathematics, vol. 531, pp. 109-115, 2010.

[4] S. Akbari, S. Alikhani, and Y.-H. Peng, "Characterization of graphs using domination polynomials," European Journal of Combinatorics, vol. 31, no. 7, pp. 1714-1724, 2010.

[5] S. Alikhani, "On the graphs with four distinct domination roots," International Journal of Computer Mathematics, vol. 88, no. 13, pp. 2717-2720, 2011.

[6] S. Alikhani and Y. H. Peng, "Introduction to domination polynomial of a graph," Ars Combinatoria. In press, http://arxiv.org/abs/0905.2251 .

[7] S. Alikhani and Y.-H. Peng, "Domination polynomials of cubic graphs of order 10," Turkish Journal of Mathematics, vol. 35, no. 3, pp. 355-366, 2011.

[8] S. Alikhani, "The domination polynomial of a graph at -1 ," Graphs and Combinatorics, 2012.

[9] K. Dohmen and P. Tittmann, "Domination reliability," Electronic Journal of Combinatorics, vol. 19, no. 1, p. 15, 2012.

[10] S. Alikhani and Y.-H. Peng, "Dominating sets of centipedes," Journal of Discrete Mathematical Sciences \& Cryptography, vol. 12, no. 4, pp. 411-428, 2009.

[11] S. Alikhani and Y.-H. Peng, "Dominating sets and domination polynomials of certain graphs. II," Opuscula Mathematica, vol. 30, no. 1, pp. 37-51, 2010.

[12] S. Alikhani and Y.-H. Peng, "Dominating sets and domination polynomials of paths," International Journal of Mathematics and Mathematical Sciences, vol. 2009, Article ID 542040, 10 pages, 2009.

[13] S. Alikhani and Y. H. Peng, "Dominating sets and domination polynomial of cycles," Global Journal of Pure and Applied Mathematics, vol. 4, no. 2, pp. 151-162, 2008.

[14] H. B. Walikar and B. D. Acharya, "Domination critical graphs," National Academy Science Letters, vol. 2, pp. 70-72, 1979.

[15] G. Chartrand and P. Zhang, Introduction to Graph Theory, McGraw Hill, Higher Education, 2005. 


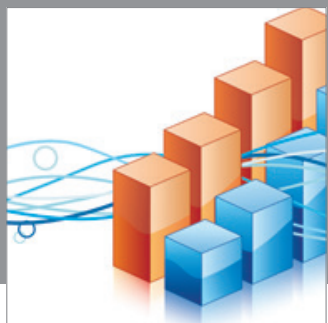

Advances in

Operations Research

mansans

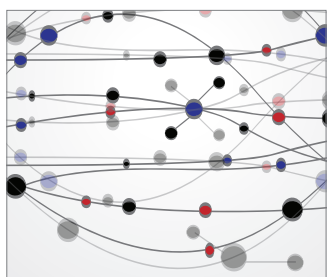

The Scientific World Journal
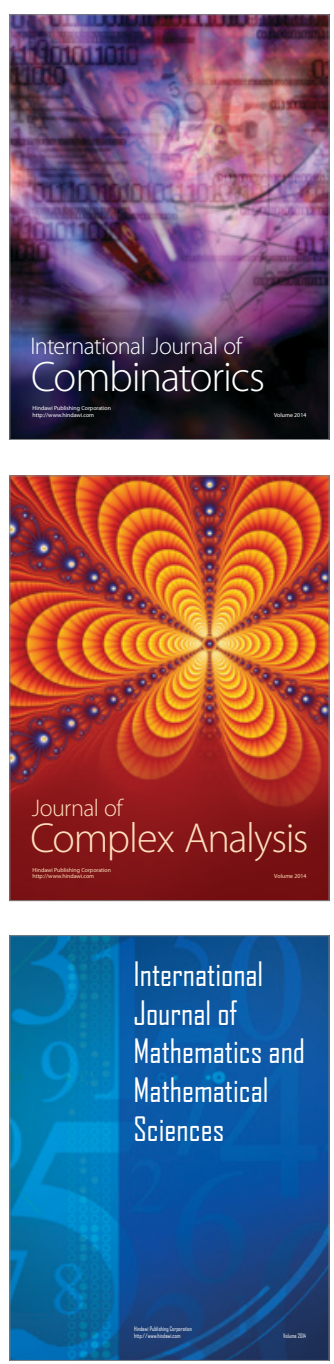
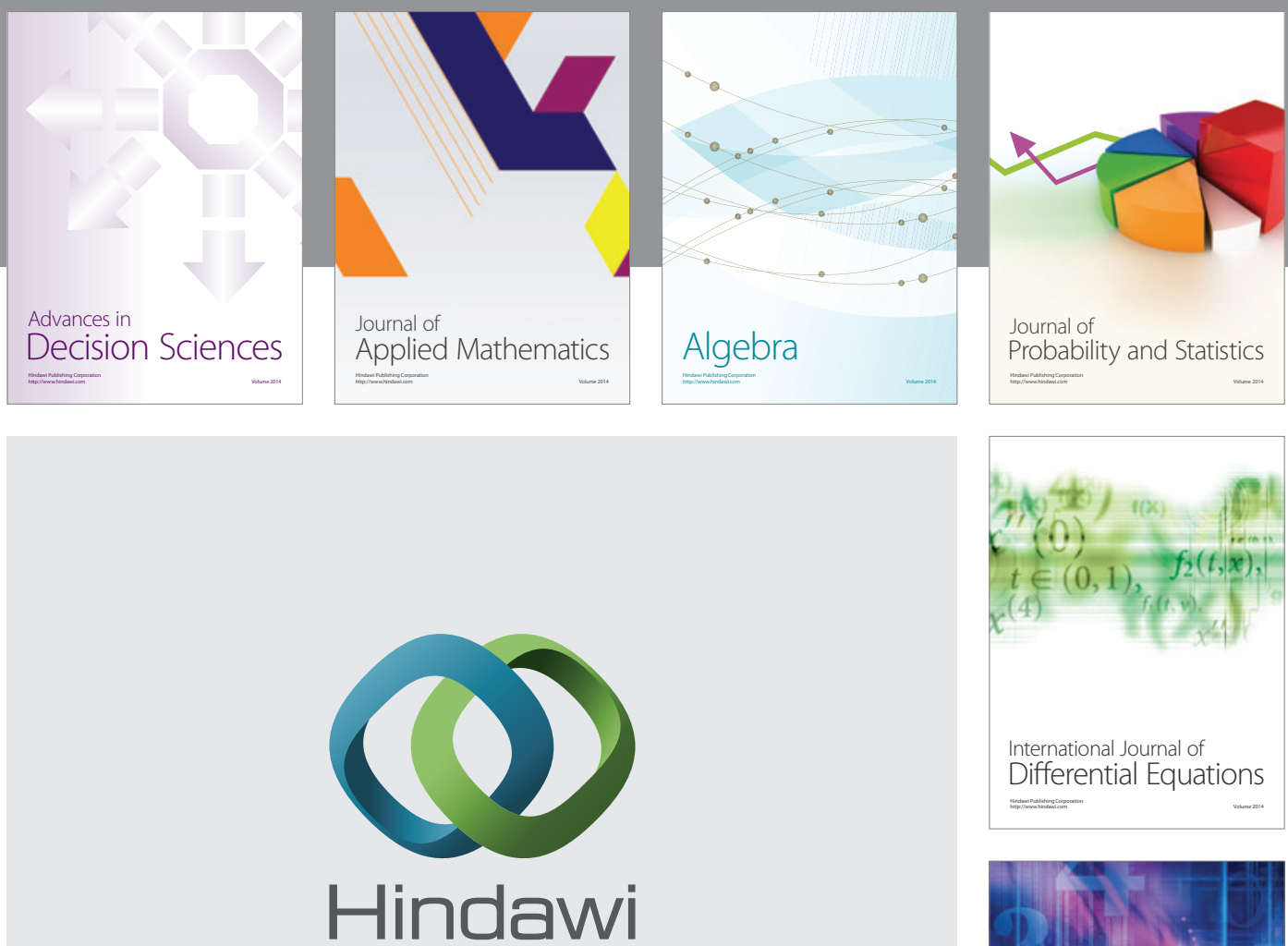

Submit your manuscripts at http://www.hindawi.com
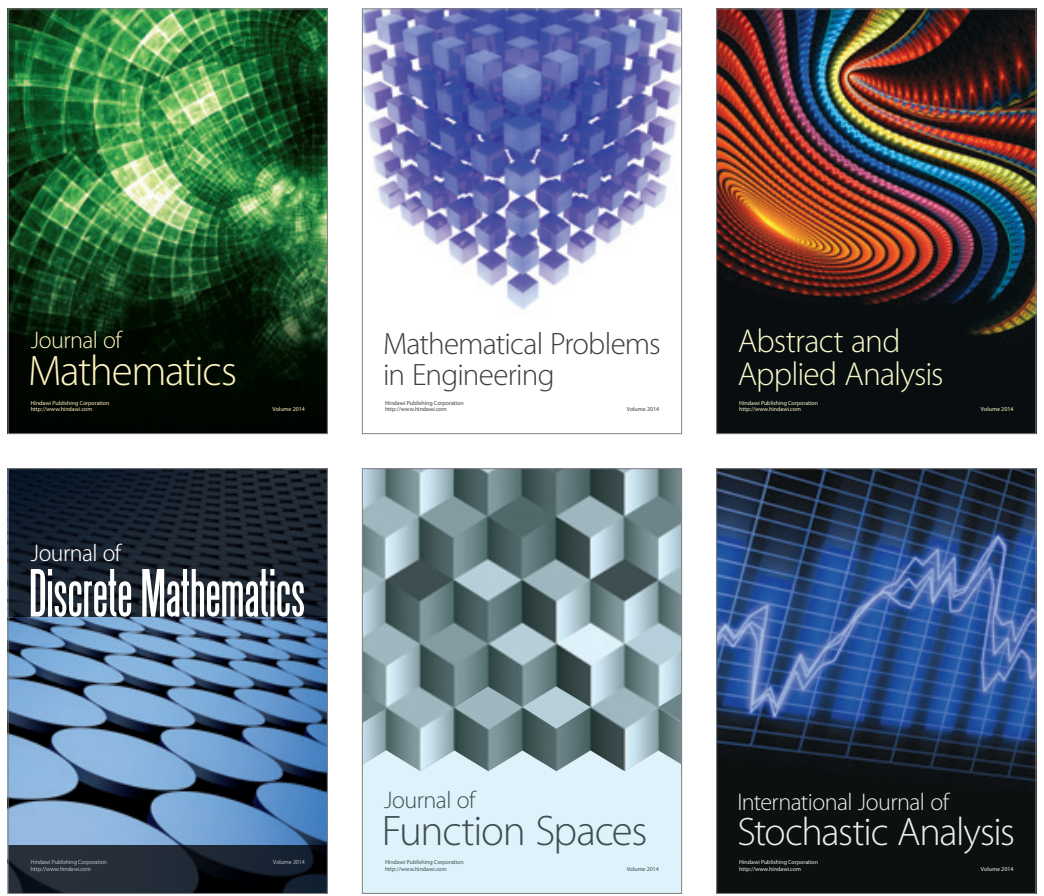

Journal of

Function Spaces

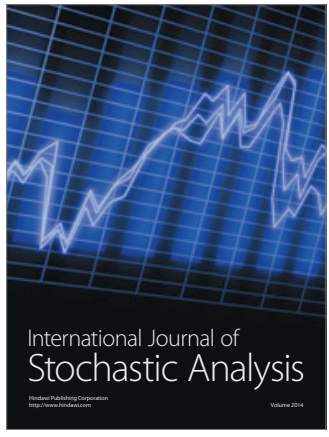

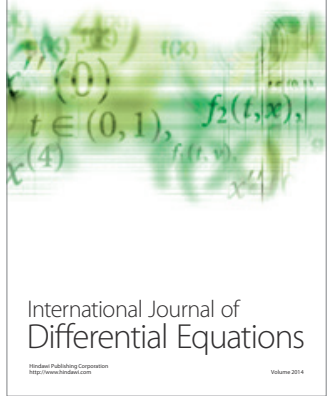
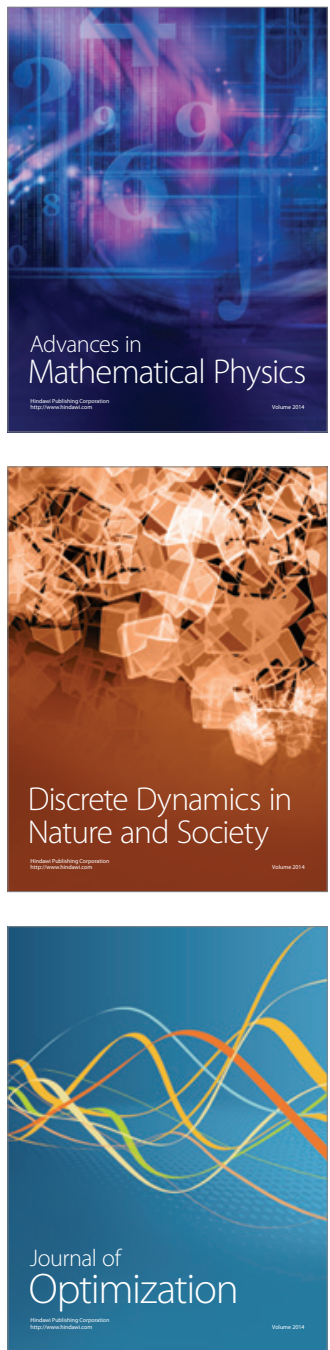